\title{
Philonsorbonne
}

13 | 2019

Année 2018-2019

\section{Le rôle du tragique dans le rapport entre l'art et la politique : Hannah Arendt contre Carl Schmitt}

\section{Katerina KAROUNIA}

\section{(2) OpenEdition}

\section{$\checkmark$ Journals}

\section{Édition électronique}

URL : https://journals.openedition.org/philonsorbonne/1094

DOI : 10.4000/philonsorbonne.1094

ISSN : 2270-7336

Éditeur

Publications de la Sorbonne

\section{Édition imprimée}

Date de publication : 30 janvier 2019

Pagination : 39-56

ISSN : 1255-183X

\section{Référence électronique}

Katerina KAROUNIA, « Le rôle du tragique dans le rapport entre l'art et la politique : Hannah Arendt contre Carl Schmitt », Philonsorbonne [En ligne], 13 | 2019, mis en ligne le 05 février 2019, consulté le 11 juin 2021. URL : http://journals.openedition.org/philonsorbonne/1094 ; DOI : https://doi.org/

10.4000/philonsorbonne.1094 


\title{
Le rôle du tragique \\ dans le rapport entre l'art et la politique : Hannah Arendt contre Carl Schmitt ${ }^{1}$
}

\author{
Katerina KAROUNIA
}

"Il est de la nature de la lumière de se refuser à la mise en lumière»

(H. Arendt, «Vérité et politique », p. 309)

\section{Introduction}

Pour Hannah Arendt les événements constituent « la texture même du domaine politique $»^{2}$. L'expérience de ce qui est n'est que la base à partir de laquelle se forment les opinions. En d'autres termes, les vérités de fait servent de «matière aux opinions $»^{3}$. Les faits ne sont ni transparents ni obscurs ; or, par référence à l'opacité de la réalité, il faut qu'ils détiennent une sorte de validité dans la sphère publique. D'après Carl Schmitt, « il faut que l'ombre de la réalité objective reste visible ${ }^{4}$. Selon Peter Riedl, Schmitt considère la réalité comme «la matière» de la tragédie ${ }^{5}$. Pour sa part, Arendt conçoit la sphère publique comme une scène de théâtre. Précisément, afin d'illustrer cette théâtralisation de l'activité politique, la

1. Cette publication fait partie de ma recherche soutenue par la Fondation hellénique pour la recherche et l'innovation (HFRI) et le Secrétariat général à la recherche et à la technologie (GSRT), dans le cadre du programme de doctorat HFRI - convention $\mathrm{n}^{\circ} 14507$.

2. H. Arendt, «Vérité et politique », dans La Crise de la culture, trad. P. Lévy, Paris, Folio, 2014, p. 294.

3. Id., p. 309.

4. C. Schmitt, Hamlet ou Hécube : l'irruption du temps dans le jeu, trad. J.-L. Besson, J. Jourdheuil, Paris, L'Arche, 1992, p. 84.

5. P. Riedl, «Time and Tragedy, Violence and Irregularity: Heiner Müller Reads Carl Schmitt », The Germanic Review: Literature, Culture, Theory, vol. 84, n 4 (2009), 353-380, p. 366 . 
tragédie constitue son exemple de choix. Y a-t-il des points de convergence entre la conception schmittienne de la tragédie et celle d'Arendt? De quelle façon la tragédie témoigne-t-elle de la réalité, pour chacun de ces deux penseurs ? Comment conçoivent-ils le rapport entre la politique et le théâtre, tout comme entre l'art et la culture ? Est-ce que leurs positions opposées à l'égard de l'art reflètent l'écart qui sépare leurs conceptions politiques ?

Afin de répondre à ces questions, on utilisera comme point de référence principal l'ouvrage de Schmitt, Hamlet ou Hécube: l'irruption du temps dans le jeu, publié en 1956. La pièce de Shakespeare Hamlet, thématisée dans Hamlet ou Hécube par Schmitt, a été présentée au public élisabéthain en 1609, lors de la première phase de la révolution anglaise. Cette époque, qui inaugure la fortune maritime de l'Angleterre, tandis qu'elle fut marquée par le conflit dogmatique entre catholicisme et protestantisme, porte aussi l'empreinte des changements profonds dus au passage de la Renaissance au baroque ${ }^{6}$, de l'ecclésiastique au politique. Le contenu tragique de la pièce est bien connu du lecteur et, comme le note Schmitt, a été commenté in extenso; il s'agit de l'histoire du «prince mélancolique, vêtu en noir », qui trouve la mort en essayant de se venger pour le meurtre de son père, roi du Danemark, commis par son oncle, Claudius.

Schmitt analyse l'intrigue de la pièce, en explorant son rapport avec le cadre historique dans lequel celle-ci a vu le jour. Il cherche ainsi à interpréter Hamlet à la lumière des faits historiques vus comme la source secrète des événements qui tissent la tragédie ; d'après lui, il s'agit d'une question qui n'a pas été traitée de manière satisfaisante. De cette façon, Schmitt propose une interprétation nouvelle, régie par l'idée que la source du « tragique » luimême s'identifie à la réalité historique.

$\mathrm{Vu}$ que Schmitt lui-même évite de formuler une définition exhaustive du tragique, le but de notre approche consiste dans son élucidation. Or, afin d'expliciter une notion, Schmitt utilise comme critère les deux contraires qui lui sont adéquats (bon-mauvais, pour la morale, ami-ennemi, pour le politique, etc.). En empruntant sa méthode, on tâchera de discerner les termes opposés qui, à notre avis, conviennent à sa façon de se référer au tragique. Ce même critère pourrait aussi nous permettre d'éclaircir le rapport entre l'esthétique et le politique. De ce fait, on va commencer par l'explicitation de la conception schmittienne de l'esthétique dans son rapport avec les méthodes interprétatives du romantisme et l'historicisme. Notons que c'est par référence à ces deux courants que Schmitt tente d'expliciter la distinction entre l'esthétique et le tragique. Tout au long de notre analyse, nous nous tournerons vers Arendt, afin d'esquisser les alternatives que ses propres approches pourraient en fournir. Aussi, certaines références à la compréhension heideggérienne du concept de l'art se révèlent être nécessaires.

6. C. Schmitt, Hamlet ou Hécube, op. cit., p. 98. 
Les idées schmittiennes sur l'art s'apparentent à divers égards aux pensées que Martin Heidegger exprime dans De l'origine de l'auvre d'art ${ }^{7}$. Tout d'abord, les deux penseurs rejettent la conception de l'art comme une reproduction de la réalité. Pour Heidegger, toute œuvre d'art - y compris la tragédie - ne constitue pas une imitation du monde, mais fait paraître quelque chose d'inhabituel ${ }^{8}$. Dans Hamlet ou Hécube, Schmitt remarque que loin d'utiliser de vrais reflets de la réalité, Shakespeare a recours à des intrusions historiques à la pièce théâtrale, qui constituent des «portes par lesquelles l'élément tragique d'une action réelle s'introduit dans le monde du jeu $\gg{ }^{9}$. Cette métaphore des portes peut être éclaircie à l'aide de la métaphore du masque et du costume théâtral que Schmitt utilise dans le même texte. C'est par le déguisement de l'acteur et à travers son masque que la réalité doit passer, afin de se trouver sur scène. Or le masque ne peut être pénétré que d'une faible lumière du réel ${ }^{10}$. Pour montrer que le personnage d'Hamlet est inspiré par le personnage historique de Jacques $\mathrm{I}^{\text {er }}$ sans en être la doublure, Schmitt écrit : « Ce serait folie de jouer Hamlet sous le masque de Jacques $\mathrm{I}^{\mathrm{er}} \gg{ }^{11}$; de toute façon, le masque tragique n'est pas assez transparent pour servir l'imitation. Ce propos schmittien s'éclaircit davantage à l'aide d'un passage qui fait partie du court texte Métaphore... La porte de Hannah Arendt. Ici, on retrouve combinées les deux métaphores schmittiennes, à savoir la porte et la lumière :

La porte est simplement tirée, n'est fermée qu'en apparence, laissant un petit rayon de lumière tomber nonchalamment l'intérieur de la pièce - on ne s'est même pas donné la peine de faire semblant de la fermer vraiment ${ }^{12}$.

Or, du fait que l'art n'imite pas son entourage, il ne faut pas conclure qu'il est complètement autonome. En effet, déjà dans Romantisme politique, Schmitt dénonce l'idée que l'art serait autosuffisant, indépendant de son contexte. Concernant les pièces shakespeariennes, il précise qu'elles ont été écrites dans le but de se produire publiquement dans un contexte historique concret $^{13}$. Dans De l'origine de l'ceuvre d'art Heidegger déclare : « De l'art

7. M. Heidegger, De l'origine de l'œuvre d'art, trad. C. Layet, Paris, Éditions Payot \& Rivages, 2014.

8. Cf. M. Heidegger, De l'origine de l'œuvre d'art, op. cit., p. 67.

9. C. Schmitt, Hamlet ou Hécube, op. cit., p. 80.

10. Ibid., p. 30. Voir surtout la traduction anglaise, où il est question d'un faint light de la réalité terrifiante : Hamlet or Hecuba, Plutarch Press, 2006, p. 18.

11. Ibid., p. 85 .

12. H. Arendt, « Métaphore... la porte », dans À travers le mur, trad. D. Meur, Paris, Payot, 2017, p. 124-5.

13. C. Schmitt, Hamlet ou Hécube, op. cit., p. 55. 
en soi, cela n'existe pas ${ }^{14}$. Du fait qu'il marque le commencement de l'existence historique d'un peuple, l'art n'est pas en soi; les œuvres artistiques signalent la grandeur du peuple en question. En plus, l'homme n'existe de manière historique que là, précisément, où l' «Être » lui-même se discerne; l'homme fait ainsi partie du peuple concret qu'il forme. C'est aussi là, au milieu de l' «étant» et du «non-étant », que l'Être s'instaure en tant qu'origine de l'œuvre artistique et essence de l'art. Selon Heidegger, les trois moments fondamentaux de l'instauration de l'Être constituent respectivement les trois «caractères» essentiels de l'art: il s'agit de 《l'offre », de la « fondation», du « commencement $»^{15}$. Or, à notre avis, le concept schmittien du tragique peut être compris à l'appui de ces trois moments. De ce fait, on va procéder à leur explication.

Commençons par la première caractéristique de l'art : l'« offre ». Outre le fait que l'œuvre d'art n'est pas une imitation, elle n'est pas non plus une représentation, parce que, selon Heidegger, «elle n'a rien qu'elle doit représenter»; exception faite de sa possibilité de représenter le non-représentable. Autrement dit, l'art offre ce qu'il fait paraître: le «non-sensible ${ }^{16}$. À titre d'exemple de ce non-sensible on peut citer la « crainte » qui, d'après Schmitt, frappait les contemporains de Shakespeare, qui « reculaient devant les réalités irrévocablement présentes» de leur époque ${ }^{17}$. D'ailleurs, le concept schmittien de la représentation se résume, justement, en ceci : rendre visible ce qui est invisible ${ }^{18}$. Aussi, inséré précautionneusement dans la pièce shakespearienne, le tabou est subsumé sous cette même catégorie du non-sensible : on se réfère ici à l'interrogation sociale sur la culpabilité de la reine Marie Stuart, pour le meurtre de son mari. L'intrusion de ce détail historique dans la pièce constitue, justement, la mise en relief du «tabou de la reine», soit comme doute concernant sa culpabilité, soit comme consentement silencieux sur cette dernière.

Il serait intéressant d'avancer ici un commentaire d'Ian Storey, concernant la poésie politique chez Arendt: il s'agit, suggère-t-il, de la vocation à dire ce qui n'est pas dit, et à créer l'espace dans lequel ce qui était ${\text { caché } s^{\prime} \text { '́lucide }}^{19}$. À première vue, Storey n'a pas tout à fait raison notamment si on prend en compte le fait que, dans "La pensée », Arendt s'oppose à l'alétheia heideggérienne, voire à «ce qui est dévoilé», en réfutant «le divorce de l'Être et de l'apparence ${ }^{20}$. Aussi Bérénice Levet signale-t-elle un passage des Cahiers arendtiens, où s'emploie la métaphore

14. M. Heidegger, De l'origine de l'œuvre d'art, op. cit., p. 89.

15. Ibid., p. 84-7.

16. M. Heidegger, De l'origine de l'œuvre d'art, op. cit. p. $70 ; 72$.

17. C. Schmitt, Hamlet ou Hécube, op. cit., p. 79.

18. C. Schmitt, Théorie de la Constitution, trad. L. Deroche, Paris, PUF, 1993, p. 347.

19. I. Storey, «The Reckless Unsaid: Arendt on Political Poetics », Critical Inquiry, vol. 41, $\mathrm{n}^{\circ} 4$ (2015), 869-892, p. 880.

20. Cf. par ex. : H. Arendt, «La pensée », dans La Vie de l'esprit, trad. L. Lotringer, Paris, PUF, 1981, p. 43. 
de l'ombre: l'ombre d'une chose apparaît de fait dans la lumière, mais l'ombre d'un acte est fabriquée par la parole ${ }^{21}$. Cela dit, la parole (voire la poésie, la littérature...) rend visible une partie méconnue de la réalité. Selon Heidegger, si l'«offre» consiste en une «expression», celle-ci n'est que «le-mettre-en-œuvre de la vérité ${ }^{22}$, conçue comme la refondation de l'alétheia.

Le deuxième moment heideggérien de l'art est la « fondation ». Suivant cette caractéristique, le concept du tragique semble enlever l'indicible, le caché, le fermé du là-historique, rien que pour le repositionner dans le monde en le fondant comme ce-qui-se ferme dans l'ouvert ${ }^{23}$. Dans Hamlet ou Hécube, Schmitt adopte une thèse semblable. Il parait que, d'après lui, la pièce shakespearienne préconise la fondation d'un mythe: la consolidation ultérieure de la «figure du vengeur $»^{24}$. Finalement, le troisième caractère de l'art est le « commencement ». Le tragique schmittien semble conditionner l'instauration d'un mythe, du fait, justement, qu'il annonce son commencement. En termes heideggériens, le tragique serait bien à l'origine de l'être-œuvre de l'étant artistique, voire de la tragédie en tant que «produit» mis en place, ou plutôt construit. Heidegger définit la «construction » qu'est la tragédie de la manière suivante : il s'agit de «l'édifice d'une œuvre littéraire, poétique, dans la langue d'un peuple ${ }^{25}$. Cet édifice est destiné à mettre en place et «à célébrer » un monde concret ; à vrai dire une connaissance vivante - en termes heideggériens - ou un savoir intègre - en termes schmittiens ${ }^{26}$. Certains éléments provenant du monde tragique sont susceptibles d'être élevés au rang d'un mythe, qui deviendra, lui-même, vivant : tel fut le cas d'Hamlet ${ }^{27}$.

\section{II.}

Dans Hamlet ou Hécube, Schmitt revisite le concept du mythe, déjà employé dans La Théorie politique du mythe de 1923. Nous allons juxtaposer les deux aspects de ce concept. En effet, Schmitt cherche une réponse à la question que lui-même se pose, à savoir : qui est aujourd'hui le porteur du grand mythe? Sorel y répond que les masses socialistes du

21. JP 2, cahier XX, novembre 1954. Cf. B. Levet, Le Musée imaginaire d'Hannah Arendt, Paris, Stock, 2011, p. 86.

22. M. Heidegger, De l'origine de l'œuvre d'art, op. cit., p. 77 ; $79 ; 86$.

23. M. Heidegger, De l'origine de l'œuvre d'art, op. cit., p. $67 ; 86$.

24. C. Schmitt, Hamlet ou Hécube, op. cit., p. 9.

25. M. Heidegger, De l'origine de l'œuvre d'art, op. cit., p. 57.

26. «C'est de la vertu d'un savoir intègre que naît l'ordre des choses humaines »; C. Schmitt, "L'ère des neutralisations et des dépolitisations", dans La Notion du politique, trad. M.-L. Steinhauser, Paris, Flammarion, 1992, p. 151.

27. Cf. C. Schmitt, Hamlet ou Hécube, op. cit., p. 13, 81-2. 
prolétariat industriel ont encore confiance en un grand mythe politique. Pour sa part, Schmitt oppose au «mythe de la lutte des classes » la plus puissante et plus fondamentale «énergie du national »: il met en avant le fascisme italien et conçoit, avec Mussolini, le mythe comme impulsion, qui, en tant que telle, puise sa vigueur dans son irrationalité. Schmitt tente par la suite de dévoiler le double fond, à la fois historico-universaliste et métaphysique, sur lequel repose, d'après lui, le mythe socialiste. Il explique que les éléments nationaux impliqués dans ce dernier viennent s'ajouter aux intérêts de classe une fois que l'«image» du bourgeois a «migré de l'Ouest vers l'Est». Il pointe ainsi l'image métaphysique à construire, laquelle se trouve à la racine du mythe politique ${ }^{28}$.

Cette image, qui présuppose un choix décisif parmi les possibles représentations du monde, est déjà politique. En effet, Schmitt assume la vision historique d'une simple - voire ni progressiste ni décadente succession de représentations du monde, d'images et de secteurs dominants. Jean-François Kervégan constate que cette succession est marquée par le « déplacement du lieu du politique ${ }^{29}$. C'est par ce déplacement constant que "l'Histoire constitue la communauté humaine générale en peuples historiquement concrets ${ }^{30}$. Cela veut dire aussi qu'après avoir été déterminé par son mythe originaire, un peuple procède à une deuxième mythification, celle de l'altérité. De caractère hostile, cette mythification négative concerne une "figure de dérision ${ }^{31}$ - telle que l'image du bourgeois susmentionnée. Or, conformément au caractère politique que revêt le portrait dérisoire de l'ennemi, ce dernier pourrait être conçu comme l'idole renversée d'un héros. Même l'«hamlétisation du vengeur », chez Hamlet, ne consiste pas en un choix purement esthétique ; la faiblesse du protagoniste est indissociable des irruptions historiques sous-entendues de la pièce $^{32}$. Celles-ci perturbent la régularité du simple jeu ${ }^{33}$, en lui opposant leur caractère sérieux.

Comme le remarque Y.-C. Zarka, le mythe schmittien peut « conférer à [...] un peuple une mission historique et un destin politique $»^{34}$. Le rôle historique du mythe se traduit ainsi en une incitation à faire son histoire. Tout d'abord, dans une lettre de 1955, adressée à Alexandre Kojève, Schmitt suggère que l'application possible d'une « hostilité sensée » conditionne la

28. Id., « La théorie politique du mythe », dans Y.-C. Zarka (éd.), Carl Schmitt ou le mythe du politique, Paris, PUF, 2009, p. 188 ; 196-7.

29. J.-F. Kervégan, Hegel, Carl Schmitt. Le politique entre spéculation et positivité, Paris, PUF, 2005, p. 106.

30. C. Schmitt, Romantisme Politique, trad. P. Linn, Paris, Libraire Valois, p. 68.

31. Id., « La théorie politique du mythe », op. cit., p. 195.

32. C. Schmitt, Hamlet ou Hécube, op. cit., p. 35. Voir aussi p. $31 ; 34 ; 36 ; 41$.

33. Ibid., p. 65 , note 19.

34. Y.-C. Zarka, «Introduction », Carl Schmitt ou le mythe du politique, op. cit., p. 11. 
faculté de «faire» l'histoire dans un ordre concret ${ }^{35}$. On comprend par là que le secteur dominant qui structure cet ordre doit être en mesure d'appliquer le critère ami-ennemi. Ce secteur reste réel, tant qu'il demeure présent. Ce qui n'est plus actuel n'est pas tout à fait réel. Une fois dépassé par la nouvelle actualité politique, il ne sera réel qu'en tant que passé actuel. En ce sens, étant " en même temps réalité et non-réalité », le passé consiste en la négation du présent ; thématiser le passé prend le sens d'ouvrir la porte à une falsification de la réalité actuelle $\mathrm{e}^{36}$.

Deux ans plus tard, et dans le même esprit, Schmitt rédige un dialogue fictif intitulé Gespräch über den neuen Raum. L'un des interlocuteurs, Herr Altmann, blâme les historiens pour leur insistance sur le récit du passé. En effet, d'après lui, « une vérité historique n'est vraie qu'une seule fois » ${ }^{37}$. Connaître l'histoire, c'est connaître le présent, affirme Schmitt dans Hamlet ou Hécube. Dans cette perspective, il soutient qu'on doit, avant tout, prendre «conscience de sa propre situation dans cette civilisation et dans cette histoire ${ }^{38}$. Le fait d'ignorer cette exigence annule la possibilité de donner un sens à sa propre sphère de la culture. Sur cette base, dans Hamlet ou Hécube Schmitt critique sévèrement l'historicisme qui, depuis le $\mathrm{XIX}^{\mathrm{e}}$ siècle, n'a fait que méconnaître cette prescription. De même, c'est par contradiction à cette entreprise historiciste, orientée vers le passé, que le caractère historique du mythe littéraire devient explicite ; précisément, Schmitt signale que se servir du passé comme source littéraire conduirait à la production de 《 contresens grotesques ${ }^{39}$. D'après lui, interpréter Hamlet en restant sur le registre historiciste ne serait qu'une impasse sans issue.

D'après la suggestion de Liisi Keedus, le propos arendtien de l'histoire se résume en une "historicité réconceptualisée »" Arendt s'oriente également contre l'historicisme. Or cette opposition ne coïncide pas avec l'antihistoricisme schmittien, hormis un point de départ commun: dans Théologie politique, Schmitt établit une analogie entre son concept de l'exception juridique et la notion du miracle. Cet ouvrage a suscité l'intérêt d'Arendt. En effet, dans son propre exemplaire, elle souligne les termes Ausnahme et Wunder $^{41}$. Pour Schmitt, la précarité de l'ordinaire se met en valeur par l'exception ${ }^{42}$. Chez Arendt, c'est la condition humaine de la

35. «Plettenberg, le 7/6/1955», Philosophie, $\mathrm{n}^{\circ} 135$ (2017) : «Alexandre Kojève face à Carl Schmitt », Paris, Les Éditions de Minuit, p. 13.

36. Cf. C. Schmitt, Romantisme Politique, op. cit., p. 79.

37. «Eine geschichtliche Wahrheit ist nur einmal wahr»; Id., «Gespräch über den neuen Raum », dans Staat, Großraum, Nomos: Arbeiten aus den Jahren 1916-1969, Berlin, Duncker \& Humblot, 1991, p. 563.

38. C. Schmitt, «L'ère des neutralisations et des dépolitisations », op. cit., p. 131.

39. C. Schmitt, Hamlet ou Hécube, op. cit., p. 85.

40. L. Keedus, The Crisis of German Historicism: the Early Political Thought of Hannah Arendt and Leo Strauss, Cambridge, Cambridge University Press, 2015, p. 126.

41. Disponible sur: http://blogs.bard.edu/arendtcollection/schmitt-carl-politische-theologie/, p. 22 ; 49. Date de dernière consultation : 28/01/2019.

42. J.-C. Monod, Penser l'ennemi, affronter l'exception, Paris, La Découverte, 2007, p. 72. 
natalité qui constitue « le miracle qui sauve le monde de la ruine normale, naturelle ${ }^{43}$. Du fait que la natalité promet le renouvellement du monde humain, elle constitue la racine ontologique de la faculté d'agir, à savoir : la capacité de commencer quelque chose de neuf que personne ne saurait prévoir. Le produit par excellence de la miraculeuse activité humaine, l'histoire, se cristallise dans l'œuvre de l'homo faber, du poète et de l'historiographe ${ }^{44}$.

Or bien que pour Arendt ce soient les récits des historiens qui «font» l'histoire ${ }^{45}$, il ne s'agit guère d'une création fantaisiste. Ce qui se joue sur la scène publique est une pièce que les acteurs créent à leur insu. Par là, l'historisation de ce qui s'est passé consiste à révéler le sens ${ }^{46}$ des événements qui tissent la narration. D'après Arendt, l'aspiration à rendre significatif un déroulement des faits discontinus est méconnue du concept moderne de l'histoire. Substituant l'explication à la compréhension, ce dernier impose à l'imprévisibilité des choses humaines ${ }^{47}$ la loi d'une causalité ex definitio fictive ${ }^{48}$. Dans Vérité et Politique, Arendt remarque qu'il s'agit d'une « illusion [...] existentielle ${ }^{49}$. Ainsi, tout en se mettant du côté de la spontanéité purement humaine que menace l'idée de la nécessité historique, elle récuse l'idée d'un processus historique qui élimine le caractère unique des événements en les absorbant. En d'autres termes, elle soutient que seuls les événements historiques éclairent leur propre passé ${ }^{50}$.

\section{III.}

Pour Schmitt, le mythe historique constitue un produit politique, du fait qu'il n'a rien à voir avec l'expansion de l'esthétique. Tout autre est le cas de la poésie lyrique qui, elle, demeure démunie d'énergie politique. Ainsi,

43. H. Arendt, Condition de l'homme moderne, trad. G. Fradier, Paris, Calmann-Lévy, 1983, p. 314 .

44. Ibid., p. 230.

45. Ibid., p. 251.

46. H. Arendt, « Isak Dinesen 1885-1963 », trad. B. Cassin, dans id., Vies politiques, Paris, Gallimard, 1974, p. 134.

47. L'histoire est dans son essence constituée d'interruptions de la continuité, constate H. Arendt (Voir « Le concept de l'histoire », dans La Crise de la culture, op. cit., p. 60), tout en précisant : l'intérêt qu'on éprouve à tort pour les processus provient de « la conviction que l'histoire est "faite" par les hommes, tout comme la nature est "faite" par Dieu » (Ibid., p. 78). 48. Cf. H. Arendt, Qu'est-ce que la politique, trad. C. Widmaier, S. Taussig, Paris, Éditions du Seuil, 2014, p. 199 ; Certes, les événements miraculeux se produisissent si souvent qu'il puisse paraître « étrange de parler encore de miracle » (H. Arendt, Qu'est-ce que la politique, op. cit., p. 188) ; mais malgré tout, l'improbabilité infinie des événements ne peut pas être méconnue.

49. H. Arendt, « Vérité et politique », op. cit., p. 309.

50. Cf. id. « Compréhension et politique », Esprit, vol. 6, n 42 (juin 1980), p. 75. 
à l'appui de sa forme sentimentale ${ }^{51}$ - propre aux concepts esthétiques -, Schmitt prend soin de la distinguer du mythe et du jeu tragique. Tandis que la source du tragique est la réalité historique, le poème lyrique n'a pas d'autre source que l'expérience subjective du poète ${ }^{52}$. Dans un contexte poétique, même un mythe ne serait rien d'autre qu'une simple hypothèse et source d'inspiration. Censé de posséder un «pouvoir poétique de création libre et souveraine $»^{53}$, un dramaturge serait prêt à reconnaître le sublime en ses propres interprétations illusoires et illimitées du monde - d'un monde devenu le simple «prétexte»d'activité artistique ${ }^{54}$ selon les directives de cet occasionnalisme romantique.

De même, malgré cet écart qui, d'après Schmitt, sépare le romantisme de la réalité, celui-ci aspire à s'insinuer ${ }^{55}$ dans tous les domaines de la vie commune, en optant pour l'esthétisation généralisée du monde. Selon Schmitt, pour définir l'essence du politique, l'adoption des critères purement moraux, économiques ou esthétiques ne peut que nous induire en erreur ${ }^{56}$. Exposé à cette méprise, le romantisme met à l'évidence la dépolitisation de la société libérale. Autrement dit, le romantisme est inapte à l'activité politique en général ${ }^{57}$. D'ailleurs, il ambitionne de composer une « musique intellectuelle sur un programme politique $»^{58}$, laquelle ne saurait ravir qu'une société bourgeoise, vouée à la consommation esthétique ${ }^{59}$. Or la bourgeoisie relativiste fait preuve d'un manque de capacité ou de volonté de concrétiser le critère du politique.

C'est dans La Notion du politique que le terme «consommation esthétique » apparaît, pour affirmer, justement, que ce manque met en relief la dégénérescence politique européenne ${ }^{60}$. Le déclin en question est dû au fait que le couple polarisé : discussion intellectuelle-compétition économique $^{61}$ l'emporte sur le politique. L'esthétique fut le dénominateur commun du moralisme et de l'économisme ${ }^{62}$. Notons que Schmitt ne méprise pas ipso facto l'admiration que soulève la forme esthétique; il ne condamne que la mise de l'esthétique au service de l'économique. L'accès à la beauté par la création de la forme esthétique est une aptitude exigeante, dans la mesure où elle est analogue à la capacité de formuler le langage d'une grande rhétorique. L'âge économique reste incapable de

51. Cf. C. Schmitt, Romantisme politique, op. cit., p. 59.

52. C. Schmitt, Hamlet ou Hécube, op. cit., p. 54.

53. Ibid., p. 51.

54. Id., Romantisme politique, op. cit., p. 33.

55. Ibid., p. 89.

56. Id., La Notion du politique, op. cit., p. 64.

57. Ibid., p. 145.

58. Id., Romantisme politique, op. cit., p. 149.

59. Ibid., p. 35.

60. Id., La Notion du politique, op. cit., p. 113.

61. Ibid., p. 117.

62. C. Schmitt, « L’ère des neutralisations... », op. cit., p. 136. 
faire usage de ce langage. Ainsi, alors qu'elle fléchit devant l'esprit romantique excessivement «musical et irrationnel », elle se livre en même temps à une impartialité muette ${ }^{63}$. Sur ce point, Schmitt coïncide avec Arendt: «La consommation et la jouissance esthétiques, si raffinées soient-elles, représentent la voie la plus sûre et la plus facile vers une emprise totale de l'économie sur la vie intellectuelle $\gg{ }^{64}$.

En effet, Arendt met l'accent sur une conception moderne erronée, laquelle impute à l'activité politique l'engendrement du philistinisme social $^{65}$. Autrement dit, elle soutient que, selon les préjugés dominants, la politique constitue une entrave à l'amélioration personnelle. En effet, le malentendu est dû à l'oubli du sens originel de la politique, ainsi qu'à la dépolitisation du domaine public, intrinsèquement liée à l'avènement de la société, l'esprit massifié de consommation, et enfin la prédominance de l'économique. Cette prépondérance de l'économique sur l'activité politique est considérée comme responsable de la perpétuation de l'avilissement culturel. À partir du constat que l'esthétique s'attelle désormais au matérialisme du philistin et de l'homme de masse, Arendt thématise le lien qui s'établit entre l'art et la politique. La motivation du philistin cultivé découle de son envie de se perfectionner, liée à son désir d'ascension sociale. Ce désir l'empêche, par exemple, de voir que "Shakespeare ou Platon pourraient avoir à lui dire des choses d'une autre importance que comment s'éduquer lui-même ${ }^{66}{ }^{6}$. Dans une société de masse, essentiellement consommatrice, les objets culturels sont usés et consommés jusqu'à ce que leur consommation devienne une fin en $\operatorname{soi}^{67}-$ comme s'ils constituaient une sorte de monnaie. Cela dit, le philistin transforme progressivement les produits de la culture, en moyens d'épanouissement personnel - comme s'il s'agissait de produits futiles servant le processus vital. Les choses culturelles deviennent alors des marchandises sociales ${ }^{68}$, vouées à l'entretien de la vie sociale. Celle-ci, à son tour, s'apparente dangereusement aux processus de la vie biologique.

Quant à la fonction de la tragédie chez Schmitt, celle-ci est conditionnée par son contexte historique que le tragique dévoile, en le présentifiant au public. C'est justement grâce au tragique que Hamlet s'est élevé au rang du mythe européen moderne. Et ceci sans être entravé par la rationalité progressiste historiciste du XVIII ${ }^{\mathrm{e}}$ siècle ni par les démythifications et dépolitisations romantiques du $\mathrm{XIX}^{\mathrm{e}}$ siècle, qui ont ouvert la voie à la privatisation moderne du domaine esthétique. Schmitt n'accepte pas qu'on

63. C. Schmitt, «Catholicisme romain et forme politique », dans C. Schmitt, La Visibilité de l'Église, Catholicisme romain et forme politique, Donoso Cortés, trad. A. Doremus, Paris, Éditions du Cerf, 2011, p. 171.

64. Id., « L’Ère des neutralisations... », op. cit., p. 136. Voir aussi Arendt ci-après.

65. H. Arendt, « La crise de la culture », dans La Crise de la culture, op. cit., p. 277.

66. Ibid., p. 260.

67. Cf. ibid., p. 270.

68. Ibid., p. 261. 
lui impute l'intention « de mettre sur le même plan l'auteur d'Hamlet et nos producteurs contemporains de films et de pièces d'actualité $\gg{ }^{69}$.

Pour sa part, Arendt se réfère au rôle des intellectuels dont elle décrit la « fonction exclusive » :

Organiser, diffuser et modifier des objets culturels en vue de persuader les masses qu'Hamlet peut être aussi divertissant que My Fair Lady et pourquoi pas, tout aussi éducatif ${ }^{70}$.

Arendt ne rend pas explicite ce qui différencie Hamlet de My Fair Lady. À vrai dire, la réponse qu'elle en aurait donnée est lisible entre les lignes citées. Si on essaie de superposer à Hamlet telle ou telle qualité philistine ce n'est qu'afin de rendre la pièce aisément digestible par les masses. En d'autres termes, cette altération rend manifeste, a contrario, qu'il s'agit d'une œuvre authentiquement culturelle; d'une œuvre dont la durée répond de son caractère fondamentalement public. Autrement dit, sa raison d'être consiste à paraître, c'est-à-dire à être là sans être aucunement utile ${ }^{71}$.

De quelle façon cette thèse se rapporte-t-elle au drame tragique? Une première réponse se trouve dans le passage de La poétique d'Aristote, qu'Arendt aime citer : «Le jeu dramatique n'est en fait qu'une imitation de l'action ${ }^{72}$. L'imitation, en tant que performance publique, rend possible l'inscription de «tel ou tel événement extraordinaire ${ }^{73}$ dans le souvenir humain. Il ne s'agit pas de construire une réplique du passé, mais de permettre aux spectateurs d'en récupérer le sens que fait naître leur propre capacité de comprendre. L'imitation revêt ainsi une acception «poḯtique ${ }^{74}$. En effet, la tragédie offrait aux spectateurs une nouvelle façon de concevoir l'importance du bios dans la polis ${ }^{75}$. De surcroît, le chant du chœur qui fait partie de la tragédie grecque, lui a fait découvrir un élément de non-imitation au sein même de l'imitation. Précisément, du fait que le chœur n'imite pas, il révèle, par son chant, l'identité narrative des héros. Il fait voir non pas ce qu'ils sont, mai qui sont-ils. Ainsi, en tant qu'élément constitutif de la pièce, la non-imitation rend la pluralité humaine présente au cœur de l'imitation. Finalement, le drame a pour unique sujet l'homme dans ses relations avec autrui; dès lors, "le théâtre est l'art politique par excellence $\gg^{76}$.

69. C. Schmitt, Hamlet ou Hécube, op. cit., p. 56.

70. H. Arendt, « La crise de la culture », op. cit., p. 266.

71. Cf. par ex. ibid., p. 279, où Arendt note que la plénitude propre aux œuvres d'art est d'apparaître.

72. H. Arendt, Condition de l'homme moderne, op. cit., p. 245 ; cf. par ex. : Aristote, Poétique, 1448a28 (cité par H. Arendt).

73. Ibid., p. 245.

74. B. Levet, Le Musée imaginaire d'Hannah Arendt, op. cit., p. 80.

75. C'est la référence d'Arendt à $C$ dipe de Sophocle, dans De la Révolution, qu'illustre ce point ; cf. H. Arendt, De la Révolution, trad. M. Berrane, Paris, Gallimard, 2012, p. 430.

76. Cf. H. Arendt, Condition de l'homme moderne, op. cit., p. 246. 
Pour sa part, Schmitt établit une analogie entre la scène théâtrale et la scène de l'action publique. Du fait que le théâtre (shakespearien) est « un morceau de présent », il se conçoit comme une partie constitutive de la réalité objective ${ }^{77}$. Cette réalité consiste aux événements qui font naître le tragique. Elle correspond soit au « savoir vivant commun » qu'est le mythe exploité dans la tragédie antique, soit aux éléments du présent historique, susceptibles de se transformer en mythe ${ }^{78}$. Inversement, la société fait partie du théâtre dans la mesure où, depuis son podium public, elle perçoit son action comme théâtrale. Afin de décrire ce double statut de la vie publique élisabéthaine et baroque, Schmitt emploie le terme «théâtralisation » ${ }^{79}$. Cette métaphore saurait-elle permettre un rapprochement de l'esthétique et du politique ? Autrement dit, serait-il opportun de supposer que, pour Schmitt, l'espace public théâtralisé, en tant qu'unité culturelle, soit analogue à une unité politique ? Et en cas de réponse affirmative, dans quel sens?

Dans Hamlet ou Hécube, il constate :

L'espace public commun qui, à chaque représentation théâtrale, englobe le poète, les acteurs et les spectateurs, ne repose pas dans le cas de la tragédie sur des règles de langage et de jeu collectivement reconnues, mais sur l'expérience vivante d'une réalité historique commune ${ }^{80}$.

Cette supériorité de l'expérience vivante historique nous fait encore penser au concept schmittien de la représentation politique : au lieu de mettre en avant l'existence physique d'un groupe, ce concept exige que ne soient propagées que les caractéristiques essentielles de l'existence commune, c'est-à-dire ses traits unificateurs les plus « élevés » ${ }^{81}$. De même, comme il est écrit dans La Théorie de la Constitution, toute unité politique obtient sa forme concrète par la réalisation des principes de l'« identité » et de la « représentation ${ }^{82}$. Au sujet de l'identité, Schmitt indique :

On pourrait se demander si la connaissance culturelle de l'histoire que l'on peut attendre des spectateurs est ou non en mesure de créer une présence commune et un espace public commun. Selon que l'on répond par oui ou non, l'histoire est une source de l'action tragique ou seulement la source littéraire d'un jeu tragique ${ }^{83}$.

77. C. Schmitt, Hamlet ou Hécube, op. cit., p. 67.

78. Ibid., p. $76 ; 80-81$.

79. Ibid., p. 66-67.

80. Ibid., p. 75.

81. Cf. id., Théorie de la Constitution, op. cit., p. 347.

82. Ibid., p. 349.

83. Id., Hamlet ou Hécube, op. cit., p. 76-77. 
Ce propos laisse entendre que la création d'un espace public commun 《 théâtralisé » ne vérifie pas seulement le fait que la tragédie tire parti de l'histoire. Certes, la « connaissance culturelle de l'histoire » n'est pas la conditio per quam de cet espace public commun $«$ inclusif $»{ }^{84}$; toutefois, elle en est bien la conditio sine qua non.

On se permet de supposer que ceux qui partagent de la même façon la conscience d'une réalité historique présentent une sorte de similitude. La connaissance commune s'avère dès lors, non pas formelle, mais substantielle - exactement comme il faut que le soit l'égalité d'une unité politique ${ }^{85}$. En fait, tout se passe comme si la théâtralisation schmittienne de la vie publique consistait en «la présentation de l'unité du tout». Il s'agit d'une phrase que Schmitt utilise pour qualifier la représentation politique ${ }^{86}$. En raison du cadre historique, concret et transitoire, qui conditionne l'approche shakespearienne du jeu tragique, Hamlet constitue pour Schmitt « la frontière mythique entre le barbare et le politique ${ }^{87}$. En effet, Schmitt constate que le jeu théâtral de l'époque élisabéthaine reste "élémentaire et barbare », puisqu'il fait partie d'une «vie non policée » ${ }^{88}$. Il s'agit d'un moment pré-étatique, caractérisé par le manque de tranquillité, de sécurité et d'ordre public ${ }^{89}$. Dans Les trois types de la pensée juridique, ces trois qualités caractérisent l'État souverain omnipotent du XVIII ${ }^{\mathrm{e}}$ siècle ${ }^{90}$, qui fût le reflet du Léviathan tout-puissant. Un Etat Léviathan, tout comme l'ultérieure " entreprise moderne » avec ses interprétations disparates de la représentation, sont peu - ou pas du tout - compétents pour la production d'images et représentations. En revanche, par son insularité, l'Angleterre en est bien capable.

Toute unité possède une forme concrète. De même, l'unité culturelle substantialisée lors d'une représentation théâtrale prendrait la forme de «l'image d'un espace public»: «Le public rassemblé dans la salle du théâtre constitue par sa présence concrète l'image d'un espace public ${ }^{91}$. $\mathrm{Du}$ fait que cet espace inclusif est imagé, il est toujours possible qu'il soit dissout, à cause de l'incapacité du public de suivre le déroulement de la représentation théâtrale. Sa préservation demeure à la discrétion du poète, qui exprime la volonté du public par une sorte de décret supra-personnel ${ }^{92}$.

84. Ibid., p. 56.

85. Cf. J.-F. Kervégan, Hegel, Schmitt. Le politique entre spéculation et positivité, op. cit., p. 318.

86. Cité dans Ibid., p. 313.

87. Ph. A. Michelbach, A. Poe, «New authority: Hamlet's politics with (and against) Carl Schmitt », Journal for Cultural Research, vol. 20, n 3 (2016), p. 6.

88. C. Schmitt, Hamlet ou Hécube, op. cit., p. 78.

89. Ibid., p. 104-5.

90. À l'exception de la «tranquillité», terme qu'il utilise ici à la place de la «paix».

Cf. Trois types de pensée juridique, trad. M. Köller, D. Séglard, Paris, PUF, 1995, p. 94.

91. C. Schmitt, Hamlet ou Hécube, op. cit., p. 56.

92. Tout comme le Führer représente l'esprit du peuple ; cf. D. Séglard, "Présentation », dans C. Schmitt, Trois types de pensée juridique, op. cit., p. 55. 
Le poète représente l'unité en tant que membre de celle-ci ${ }^{93}$. Il n'est pas en position de méconnaître le "(fort) noyau de réalité » ${ }^{94}$ ni le fait que son public ait conscience dudit noyau. À première vue, on pourrait dire que cet espace public fictif, formé par les spectateurs qui sont présents dans la salle du théâtre, évoque le concept arendtien du « monde ».

\section{IV.}

Dans Hamlet ou Hécube, tout se passe comme si, après l'intrusion du temps historique dans le temps théâtral, venait l'ingérence de l'espace théâtral dans l'espace public ${ }^{95}$. Chez Arendt, le théâtre est, certes, une transposition imitative du réel; mais le monde - quand le terme ne signale pas le cadre matériel conditionnant l'activité humaine - se forme directement dans la sphère publique : il s'agit de l'espace intermédiaire qui, à la fois, rassemble et sépare les personnes qui y sont présentes ${ }^{96}$. Cette différence, qui peut être qualifiée d'essentielle, on va l'articuler en trois moments.

Primo, les acteurs qui paraissent sous la lumière publique sont pour Arendt des personnes publiques, dont le paraitre constitue l'être. En d'autres termes, au lieu de s'exhiber ou d'exposer ce qu'ils sont, ils exposent leur persona. C'est ainsi que, d'un point de vue arendtien, la théâtralisation que Schmitt semble hisser en représentation ne serait qu'une représentation dégénérée en spectacle. D'ailleurs, Schmitt lui-même utilise le terme « spectacle» pour qualifier l' « agir en public » ${ }^{97}$.

Secundo, les êtres humains sont potentiellement confrontés au jamais$v u$; faute de critères préétablis, le particulier, étant sans précédent, ne peut pas être subsumé sous un concept donné. Une telle situation requiert l'actualisation de la faculté de juger, qui est alors beaucoup plus proche « de la capacité de distinguer que de la capacité [...] de subsumer ${ }^{98}$. Certes, ce type de jugement direct, ou plutôt cette décision, est, en un sens, de caractère subjectif; car la personne qui, sur la base d'une expérience inédite, prend

93. C. Schmitt, Hamlet ou Hécube, op. cit., p. 56.

94. Ibid., p. 71.

95. Dans l'Annexe II de Truth and Method, London - New York, Continuum, 2004, p. 499, Gadamer suggère que Schmitt omet de reconnaître l'irruption du jeu dans le temps et, par la suite, le caractère innovant du mythe et la nature du jeu artistique. Nous pensons que Schmitt accorde la capacité d'incursion dans le réel au théâtre de l'époque élisabéthaine ; et que cette irruption inversée se traduit par la formation d'un espace public à l'image de l'espace théâtrale.

96. H. Arendt, Qu'est-ce que la politique, op. cit., p. 179.

97. «Agir en public c'était agir sur une scène et c'était donc du spectacle »; C. Schmitt, Hamlet ou Hécube, op. cit., p. 66.

98. H. Arendt, Qu'est-ce que la politique, op. cit., p. 175. 
une « décision sur la sorte d'action à entreprendre, la façon de voir le monde à l'avenir et les choses qui y doivent apparaître », ne perçoit qu'un certain aspect du monde, en fonction de la position qu'il y occupe. Cependant, il reste libre de se mouvoir dans ce monde qui est un «datum objectif» commun $^{99}$. Cela ouvre la voie à l'interaction entre les hommes, dans des conditions d'isonomie. En fin du compte, décider ne consiste guère, pour Arendt, à ordonner. Pareille à une scène de théâtre, la sphère publique - sans souverain et représentant ultime - produit une pièce dont le père-créateur n'existe pas non plus.

Tertio, pour Schmitt, assis dans la salle du théâtre le public fait partie d'une unité ; ce qui se passe sur la scène les participants l'aperçoivent du point de vue de l'unité qu'ils forment: "Même les rêves que l'auteur dramatique mêle à son jeu doivent pouvoir être rêvés conjointement par les spectateurs $\gg{ }^{100}$. Afin de tracer la différence structurelle entre la conception schmittienne et la pensée arendtienne, il faut prêter son attention au terme « conjointement ». Dans Liberté et politique, Hannah Arendt soutient que la virtuosité, dont fait preuve une performance théâtrale, dépend de la façon dont la conçoivent les spectateurs ${ }^{101}$. Chacun d'entre eux n'en a pas une expérience identique. Il en devient explicite que le principe de l'identité, inéluctable pour la cohérence de la thèse schmittienne, est étranger au monde arendtien. Arendt définit la salle du théâtre à l'instar du monde public et vice versa. En ce sens, à l'opposé d'une unité absolue, les spectateurs constituent une pluralité d'êtres uniques. Cela dit, de façon qui évoque la constitution du monde arendtien, chaque individu envisage de son point de vue et partage ainsi avec les autres la réalité façonnée que la pièce met sous les yeux. Or ce qui importe à Arendt est qu'il s'agit d'un individu désintéressé.

Cela dit, loin de rêver les mêmes rêves, les spectateurs, grâce à leur sens commun, sont à même d'énoncer des jugements qui sont politiques dans la mesure où ils sont esthétiques ${ }^{102}$ : «L'originalité de l'artiste (ou la nouveauté de l'acteur) est liée à sa capacité de se faire comprendre des non-artistes (ou des non-acteurs) ${ }^{103}$. Afin que la beauté des objets soit perçue, le seul système sensoriel ne suffit pas. Car la distinction entre le beau et le laid dépend du jugement esthétique des spectateurs. Paradoxalement, ce jugement qui aspire à l'objectivité demeure fondé sur le goût ; évidemment, il s'agit d'une affaire « surprenante », parce le goût est, certes, un sens privé. Cependant, il n'est pas exclusivement subjectif, vu que le point de référence d'un jugement est la réalité ; sa condition ultime est l'imagination. À l'instar du goût consistant en un «contentement oisif», l'imagination fait « abstraction des bornes », en ce qu'elle permet le déplacement du sujet

99. Id., La Crise de la culture, op. cit., p. 284-285.

100. C. Schmitt, Hamlet ou Hécube, op. cit., p. 57.

101. Cette dépendance réciproque entre les acteurs et les spectateurs fonde l'analogie entre le lieu d'expérience théâtrale, et l'espace politiquement organisé.

102. Cf. H. Arendt, « La crise de la culture », op. cit., p. 284.

103. Id., « Le juger », Vie de l'esprit, op. cit., p. 554. 
afin qu'il puisse inspecter le plus grand nombre de points de vue : existants, possibles ou éventuels ${ }^{104}$. C'est enfin grâce à son caractère " collectif » ${ }^{105}$ que le goût a le pouvoir d' «humaniser» le beau et le monde. En effet, humaniser le beau veut dire : faire en sorte qu'il devienne l'objet de l'esprit élargi. De même, le monde s'humanise en tant qu'il sauvegarde sa capacité de faire accueil à la pluralité des êtres qui sont les mêmes et différents et qui agissent en concert. On est humaniste quand on exerce librement le goût. Et puisque le goût est une faculté, à la fois, esthétique et proprement politique, être humaniste signifie qu'on s'élève au-dessus des conflits entre l'homme d'État et l'artiste ${ }^{106}$.

Or, selon Arendt, « la culture et la politique s'entr'appartiennent ${ }^{107}$; en d'autres termes, "la culture indique que l'art et la politique [...] sont liés, et même en mutuelle dépendance ${ }^{108}$. Certes, Arendt pense que la vie politique ne se transforme en œuvre d'art que par le théâtre ${ }^{109}$. Il s'agit de deux phénomènes mondains, qui, en tant que tels, nécessitent un espace public où être vus. Aussi, contrairement au caractère éphémère de l'action, la culture, par la durée de ses produits, sauvegarde la mémoire du monde incessamment renouvelé, en l'enrichissant des histoires humaines qu'elle préserve. Autrement dit, l'art sauvegarde les hauts faits humains en les réifiant: «Les œuvres d'art sont des objets de pensée, mais elles n'en sont pas moins des objets $»{ }^{110}$. Or il s'agit des artifices qui contrairement aux choses fabriquées demeurent étrangers à l'idéal utilitaire ${ }^{111}$. De toute façon, si la construction du monde artificiel garantit la permanence de l'habitation humaine sur terre, la réification des histoires par leur transformation en œuvre d'art rend possible l'immortalisation des mortels. L'immortalité humaine est médiatisée par la beauté ; celle-ci consiste précisément en « la gloire radieuse par laquelle une immortalité potentielle est rendue manifeste dans le monde ${ }^{112}$. Ainsi, la beauté lie la politique avec le critère esthétique. C'est à l'aide de ce critère précisément, qu'elle revêt un caractère d'exemplarité. Autrement dit, c'est en réifiant les hauts faits des hommes qui se déroulent sur la scène publique, que l'art opère leur immortalisation. De cette façon, devenus des produits de la culture, ils préservent le caractère éminemment humain du monde dont ils font, désormais, partie intégrale. En revanche, il semble que l'ingérence de l'esthétique dans l'espace public schmittien ne se révèle utile qu'à condition d'attester du caractère homogénéisateur de cet espace.

104. Ibid., p. $547-8 ; 551 ; 555$.

105. Ibid., p. 562.

106. Id., La Crise de la culture, op. cit., p. $286 ; 288$.

107. Ibid., p. 285.

108. Ibid., p. 279.

109. Id., Condition de l'homme moderne, op. cit., p. 246.

110. Cf. Ibid., p. $224 ; 229$.

111. Cf. Ibid., p. 230.

112. Id., La crise de la culture, op. cit., p. 279. 


\section{Conclusion}

Dans La Notion du politique, Schmitt soutient que la distinction entre le beau et le laid ne doit pas être confondue avec la discrimination politique entre l'ami et l'ennemi. La deuxième est conçue comme un "principe d'identification qui a valeur de critère $\gg{ }^{113}$, tandis que la première relève du domaine de l'esthétique. Pourtant, ce dernier est déjà politisé, qualifié de non-politique d'après une décision politique ${ }^{114}$. Pour sa part, Schmitt affirme que la distinction politique peut être, à son tour, esthétisée. Sauf que la force de la réalité historique est supérieure à la force de «n'importe quelle esthétique $»^{115}$. Ainsi, l'intrusion du réel dans le drame demeure un concept anti-esthétique $^{116}$, tout en contribuant à l'esthétisation instrumentale du politique.

De prime abord, le tragique parait être le moyen de réaliser cette intrusion historique. Nous pouvons maintenant revenir à notre point de départ, inspiré par la pensée heideggérienne. Conformément à la pensée schmittienne, le tragique sert d'élément constitutif d'une œuvre artistique, pour les raisons suivantes: a) il "offre» au public un sentiment de contiguïté, grâce à son ancrage dans le réel; b) il « fonde » le caractère sérieux de l'œuvre en question sur l'actualité historique ; c) il « commence » un processus, censé aboutir à l'instauration d'un nouveau mythe vivant. On peut en déduire que le tragique conditionne l'aperçu, l'approbation et la transmission de l'art et de l'histoire. Cela, puisqu'à la subjectivité artistique, il objecte l'objectivité historique. Chez Arendt, la thématique de l' $\dot{\alpha} \theta \alpha v \alpha \tau i \zeta \varepsilon v v^{117}$ constitue le fil rouge entre la politique et l'esthétique. En tant que persistance d'un événement historique au sein du souvenir humain, elle a recours à l'élément tragique : la fin de la révolution hongroise a été marquée par le deuil public des femmes dans les rues de Budapest. Selon Arendt, cet acte a été le dernier geste révolutionnaire, d'un point de vue politique. Il a exprimé idéalement et l'essence tragique et la sublimité de la révolution. «Qui pourrait l'oublier? » ${ }^{118}$, se demande-t-elle.

À y regarder de plus près, bien que Schmitt lui-même ne le dise pas explicitement, on peut soutenir que sa notion du tragique se spécifie à l'aide de la distinction réel-fictif. En ce sens, le tragique lui-même devient, à son tour, le critère qui nous permet de juger de l'authenticité ou de l'intensité de

113. C. Schmitt, La Notion du politique, op. cit., p. 64.

114. Cf. id., Théologie politique, op. cit., p. 12.

115. Id., Hamlet ou Hécube, op. cit., p. 48.

116. H. Cho, «Humor or Dying Voice - Hamlet between Walter Benjamin and Carl Schmitt », The Germanic Review: Literature, Culture, Theory, vol. 91, n 3 (2016), 258-276, p. 264.

117. H. Arendt, « Le concept de l'histoire », op. cit., cf. par ex. p. 101.

118. Id., " Totalitarian Imperialism: reflections on the Hungarian revolution », The Journal of Politics, vol. 20, n 2 (1958), 5-43, p. $5 ; 7$. 
la connexion entre l'élément sérieux de l'histoire et l'élément ludique de l'art. Autrement dit, à l'aide du critère du tragique on pourrait trancher, dans chaque cas précis, sur la compatibilité du politique avec l'esthétique. Pour utiliser contre Schmitt sa propre ironie, cette compatibilité se résumerait au degré auquel la beauté purement esthétique a été troublée, et la valeur propre de la forme artistique a été dégradée ${ }^{19}$; ou inversement, d'après le commentaire de David Pan, au degré auquel l'art est devenu «fonctionnel pour le système politique ${ }^{120}$.

Arendt met en avant la complémentarité qui caractérise les fonctions politiques du poète et de l'historien : premièrement, la catharsis, qui facilite l'action par la « purgation des passions »; deuxièmement, la bonne foi, qui témoigne de la réalité ${ }^{121}$. Leur interdépendance reflète le lien qui se forme entre l'action et la parole au sein de la sphère politique. Celle-ci est esthétisée a priori. De même, Arendt se sert de la culture et de l'œuvre d'art pour mettre au jour l'importance politique de la mentalité élargie. En revanche, d'après Victoria Kahn ${ }^{122}$, Schmitt reproche à la culture de contrer, non pas la contemplation désintéressée, mais le conflit existentiel de l'espace public. M. Michelbach et A. Poe précisent que l'esthétisation schmittienne du politique se traduit en représentation esthétique de l'autorité souveraine $^{123}$. Il s'ensuit que la façon dont Arendt conçoit la théâtralisation de la vie publique se trouve aux antipodes de la pensée schmitienne. Qu'il se trouve sur la scène, devant elle, ou même dans les coulisses, chacun pourrait se faire « le représentant de qui que ce soit d'autre ${ }^{124}$; et ceci, pourvu qu'il soit capable de mettre en œuvre sa faculté de juger.

119. C. Schmitt, Hamlet ou Hécube, op. cit., p. 51.

120. Cf. D. Pan, «Political Aesthetics: Carl Schmitt on Hamlet», Telos, vol. 72 (1987), 153-9, p. 155.

121. H. Arendt, «Vérité et politique », op. cit., p. 334.

122. V. Kahn, «Hamlet or Hecuba: Carl Schmitt's Decision», Representations, vol. 83, $\mathrm{n}^{\circ} 1$ (2003), p. 67-96, p. 69.

123. Cf. P. Michelbach, A. Poe, «New authority: Hamlet's politics with (and against) Carl Schmitt », op. cit., p. 6.

124. H. Arendt, «Vérité et politique », op. cit., p. 308. 